\title{
Are the Sites of Endocardial Origin of the Left Ventricular Papillary Muscles Primary Determinants of the Morphology of the Normal QRS Complex?
}

\author{
AMC Robinson ${ }^{1}$, CW Olson ${ }^{2}$, G Wagner ${ }^{3}$, RH Selvester ${ }^{4}$, \\ J Foster $^{5}$, T Steedman ${ }^{6}$ \\ ${ }^{1}$ Uinversity of Glasgow, Glasgow, Scotland \\ ${ }^{2}$ ECG-TECH Corp Huntington, New York, USA \\ ${ }^{3}$ Duke University Medical Center, Durham, North Carolina, USA \\ ${ }^{4}$ University of Southern California, Long Beach, California, USA \\ ${ }^{5}$ Glasgow Cardiac Magnetic Resonance Unit, Glasgow, Scotland \\ ${ }^{6}$ Glasgow Cardiac Magnetic Resonance Unit, Glasgow, Scotland
}

\begin{abstract}
There is extensive variation in the appearance of the QRS complex of a standard 12 lead ECG of normal subjects. This study tests the hypothesis that a primary determinant of the morphology of the normal QRS complex of the left ventricle is papillary muscles origin. Magnetic resonance imaging was used to determine the site of endocardial insertion of the papillary muscles. The papillary muscle data was then inputted into a schematic, which was used to guide a simulation of the left ventricular activation. A simulated ECG and vectorcardiogram (VCG) were produced. The 10 subjects used were healthy with no history of cardiac disease. The simulated and original ECGs were compared for each subject; the frontal electrical axis was calculated and compared. VCGs produced for each individual were analyzed and the effect of the papillary muscle position on VCG morphology analyzed. It was found that the simulated and original ECGs for each subject had similar QRS waveforms that showed normal morphologies. The frontal axis had a good correlation but no concordance and the VCG showed the closer the papillary muscle origins to the septum the wider the VCG spread.
\end{abstract}

\section{Introduction}

The left ventricle has two papillary muscles form part of the apparatus of the mitral valve; they aid the closure of the valve leaflets prior to ventricular contraction allowing efficient forward ejection of the left ventricle volume. The papillary muscles insert indirectly into the endocardium via the trabeculae carena [1] and indirectly in the valve leaflets via the chorda tendinea. The insertion site of the muscles vary, the ventricle can be divided into 3 segments with the majority of papillary muscle attaching in the mid-third of the ventricle [2,3]. In the left ventricle, the left bundle branch fans out over the septal wall, the anterior and posterior borders of the fan are limited by the attachments of the mitral papillary muscles [3].

Magnetic resonance imaging has shown the ability to image soft tissue structure within the body in great detail. It has the ability to locate the position of the papillary muscles on the endocardium as well as delineate their cross-section and length. This information can then be used for the basis for a simulation of the ECG and VCG, in which the location for the initial excitation of the left ventricle can be associated with the physical location of the papillary muscles.

To generate a realistic ECG of the QRS complex a computer program [4] was used which allowed selection of the initial excitation points on the left ventricle. The program shows a 3-D display of cardiac excitation to illustrate the process of generating cardiac electrical signals. The display shows the projection of the $3 \mathrm{D}$ vector onto the separate transverse, frontal and sagittal planes. 12 lead signals are shown on the same display so that they can be related to the total vector in time sequence. Thus the process of how the ECG signal is generated from dipole surfaces starting on the endocardium and projecting to the epicardium is directly related to its method of measurement. To achieve greater accuracy in the translation of the $\mathrm{X}, \mathrm{Y}, \mathrm{Z}$ signals to the 12 lead, a geometric transform was used.

\section{Methods}

There is extensive variation in the appearance of the QRS complex of normal ECG. Multiple factors influence the body surface morphology of these waveforms and this study tests the hypothesis that a primary determinate in 
the QRS morphology is the position of endocardial origins of the left ventricular papillary muscles.

The study population consisted of 10 healthy volunteers with no history of cardiac conditions, they were mixed age and sex and all white caucasion.

All subjects underwent a 1.5-Teslar cardiac magnetic resonance (MRI) scan. Sequential short axis slice through the left ventricle were acquired; $4 \mathrm{~mm}$ thick and with $1 \mathrm{~mm}$ between slices. All images were performed with breath holds. Each image was analysed independently using a MRI analysis program (Cardio Warp). Analysis was preformed 3 times by 1 observer, to investigate the reproducibility of the analysis.

1. Angels of the papillary muscle was calculated in each short axis slice.

2. Insertion point of the papillary muscle was calculated as a percentage of the total length of the left ventricle in the long axis slice.

Validation of the MRI method was performed to find out the observer variability and to test the reproducibility of the method.

An ECG technician using a Phillips ECG recorder took the digital ECG recordings after each MRI scan.

The data from 2 subjects were sent to Olson, he used the papillary muscle insertion sites as activation sites for simulated ventricular contraction and produce a QRS complex but found Q waves in V1,V2 and V3. Based on these results the excitation sites were altered; site were moved basally by $1 \mathrm{~cm}$. This removed the $\mathrm{Q}$ waves but QRS loop was moved posteriorly, 3 further sites of excitation were introduced on the septum. All remaining subject had ECGs generated based on all 3 alterations and a geometric transform was used to converted $\mathrm{X}, \mathrm{Y}, \mathrm{Z}$ coordinated to 12 lead ECG. The simulation was altered to take in to account the individual anatomical axis, which was calculated from the MRI data.

Subject 7 was unusual and required further study. The computer generated ECG did not have Q waves but the standard did, it was suggested that this may be due to a long thin chest which may have lead to different positioning of the heart with in the thorax. Changing the position of the electrodes to one interspace lower caused the $\mathrm{Q}$ waves to disappear.

Dr Wagner who acted as a core lab carried out the analysis of the ECG. The frontal electrical axis was calculated by Dr Wagner who was blinded to the identity of the individual.

\section{Results}

Statistical analysis was carried out to see how reproducible the MRI method was.
Table 1: Pearson correlation by reading comparison

\begin{tabular}{llll}
\hline & Reading & & \\
\hline Variables & 1 vs. 2 & 1 vs. 3 & 2 vs. 3 \\
Anterior angle & 0.992 & 0.994 & 0.996 \\
& $(\mathrm{p}<0.0001)$ & $(\mathrm{p}<0.0001)$ & $(\mathrm{p}<0.0001)$ \\
Posterior angle & 0.963 & 0.956 & 0.984 \\
& $(\mathrm{p}<0.0001)$ & $(\mathrm{p}<0.0001)$ & $(\mathrm{p}<0.0001)$ \\
\hline
\end{tabular}

This table shows the variability in the results of the MRI analysis of papillary angles when comparing the $1^{\text {st }}$ MRI analysis with the $2^{\text {nd }}$ analysis, the $1^{\text {st }}$ MRI analysis compared with the $3^{\text {rd }}$ analysis and the $2^{\text {nd }}$ MRI analysis compared with the $3^{\text {rd }}$ analysis. The values given show Pearson correlation values and the probability coefficient (p-values). Pearson correlation coefficient shows correlations were very high indicating high levels of statistical significance. Values were adjusted for the fact that some subjects had more readings than others.

Table 2: Spearman rank correlation by reading comparison $(\mathrm{n}=10)$

\begin{tabular}{llll}
\hline & Reading & & \\
\hline Variables & 1 vs. 2 & 1 vs. 3 & 2 vs. 3 \\
Longitudinal & 0.867 & 0.915 & 0.976 \\
length & $(\mathrm{p}=0.001)$ & $(\mathrm{p}<0.0001)$ & $(\mathrm{p}<0.0001)$ \\
Apex & 0.903 & 0.976 & 0.952 \\
insertion & $(\mathrm{p}<0.0001)$ & $(\mathrm{p}<0.0001)$ & $(\mathrm{p}<0.0001)$ \\
Valve & 0.879 & 0.888 & 0.997 \\
insertion & $(\mathrm{p}=0.001)$ & $(\mathrm{p}=0.001)$ & $(\mathrm{p}<0.0001)$ \\
$\%$ of total & 0.939 & 0.394 & 0.321 \\
length & $(\mathrm{p}<0.0001)$ & $(\mathrm{p}=0.33)$ & $(\mathrm{p}=0.36)$ \\
$\%$ error & -0.334 & 0.784 & -0.442 \\
& $(\mathrm{p}=0.34)$ & $(\mathrm{p}=0.007)$ & $(\mathrm{p}=0.20)$ \\
\hline
\end{tabular}

This table shows the variability in the results of the MRI analysis of the left ventricle length, apex to papillary insertion length, valve to papillary muscle insertion length and the percentage of the total length along with percentage error. The values given show the Spearman rank coefficient and the probability coefficient (p-valve). Percentage of total length is the point at which the papillary muscle as a percentage of the left ventricle length. Spearman rank correlation coefficient was used to examine the association between readings. It was use because of the small sample number, 10. Spearman values were high for length, apex to base, but readings were less reliable for percentage total length and percentage error. 
Fig 1. Comparison of original and standard ECGs

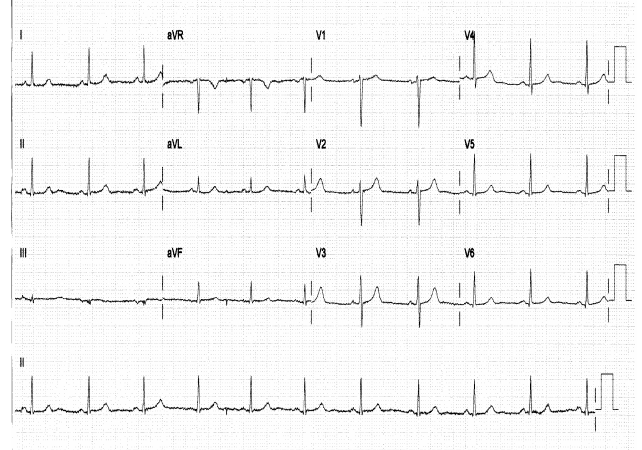

\section{Original ECG}

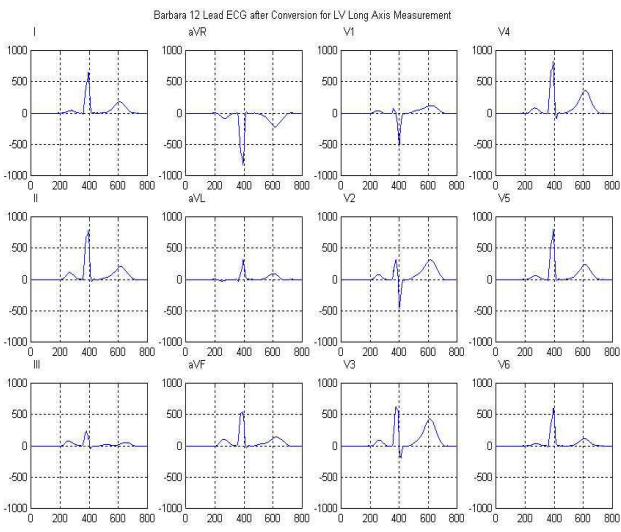

Simulated ECG

Fig 2: Comparison of Frontal axis angles

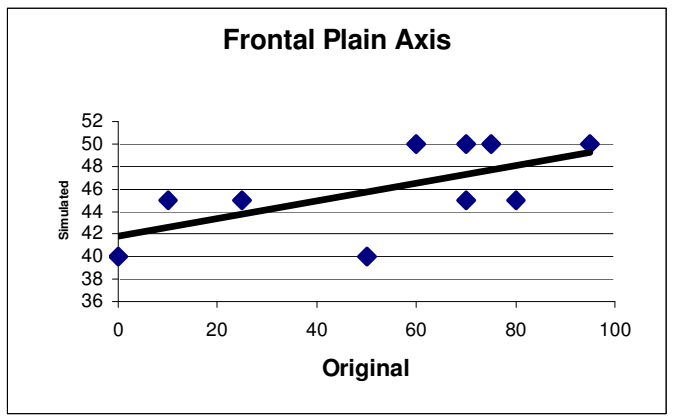

Fig 2.1. Correlation between original and simulated frontal plain axis.

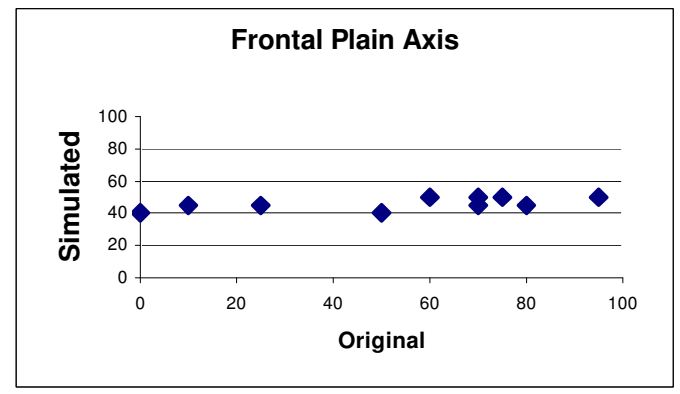

Fig. 2.2. Concordance between original and simulated frontal plain axis.

Figure 3.

VCG of two patients with very different papillary muscle position.

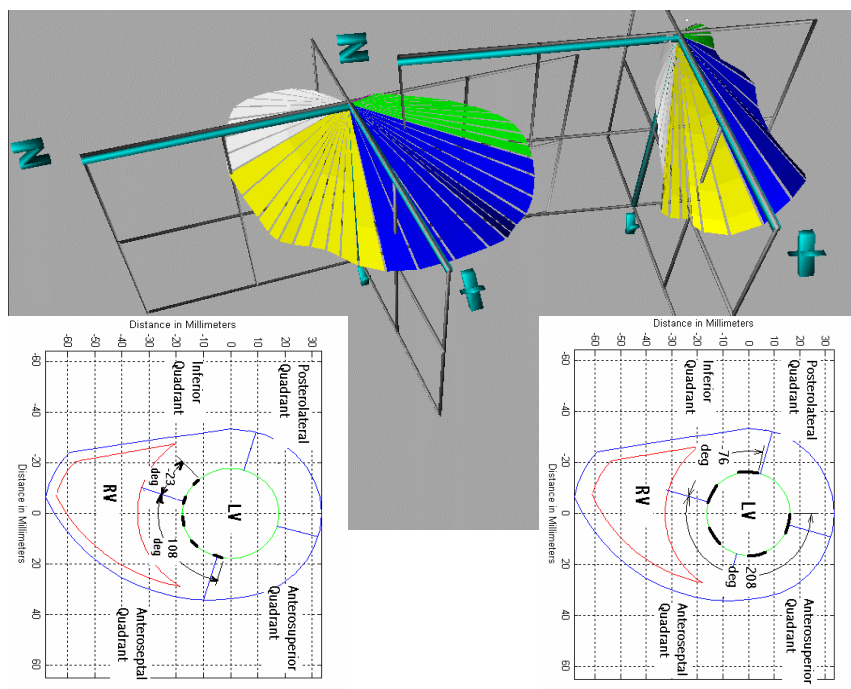

The method of MRI analysis used was reliability for the angle of insertion with Pearson correlation values high for both posterior and anterior papillary muscles. $\mathrm{P}$ values of $<0.0001$ were produced. Spearman correlation coefficients were worked out for length measurements. The reliability for the longitudinal length of the left ventricle had a $\mathrm{p}$ valve of 0.001 . The apex to insertion $\mathrm{p}$ valve was $<0.0001$ showing good reliability. The valve to insertion had a $\mathrm{p}$ value of 0.001 which was also highly reproducible data. The percentage total length and percentage error had low reproducibility this would be expected due to compounding variables.

Nine of the volunteers that were involved in the study were used in the final analysis. Subject 7 was excluded because he had a standard ECG that was of concern due to the presence of $\mathrm{Q}$ waves in V1, V2, V3 and presence of abnormally high $\mathrm{T}$ waves. It was hypothesised that these 
abnormal Qs were caused by torso morphology, a long thin body compared to height. The heart may have been positioned higher or lower than expected in the thoracic cavity. This would have meant that the chest leads for the ECG would not have been over the correct area of the heart to produce a 'normal' ECG, for a healthy patient. Two further ECGs were taken, one with electrodes one intercostals space higher, and one with electrodes one intercostals space lower than the standard positions. V1 and V2 lost their Q wave in the one inter-space lower ECG, confirming the lower position of the heart in this individual. It was decided that this subject would not be included in the final analysis of the results because of the change to the protocol (his results are shown for one inter-space lower but not included in the analysis).

Subject 9 also has a similarly long thin chest compared to height and it was hypothesised that this abnormal ECG was caused by the chest morphology. It was decided further ECGs should be performed; this has not been done due to the volunteer being unavailable but will be carried out as soon as possible.

Variations in homogeneities of the torso will affect the ECG. The standard ECG is taken from surface measurements where as the computer generated ECG in produced from anatomical data, so is not affected by these homogeneities but may be affected by anatomical variation in the papillary muscle form and insertion.

In figure 1 it can been seen that the simulation produces a ECG with morphology similar to the original with normal QRS morphologies.

Figure 2 the frontal plane shows good correlation but there is no concordance between the original and simulated ECG.

Figure 3 shows the VCGs and the left ventricle crosssections with papillary muscle positions shown. The closer the papillary muscles are to the septum the wider the vector cardiogram in the horizontal plane. The longer the excitation takes to spread over the left ventricle, due to the larger area that has to be excited by intra myocardial spread. The closer the papillary muscles to the septum the narrow the purkinje fan is.

This study is the preliminary part of what is hoped to become a larger piece of work. A small study sample was used, (10 patients) to allow from the development of the MRI method, the MRI analysis method and computer ECG generation method.

The information that was inputted into the ECG simulation program was the position of the papillary muscles in terms of distance from bases and apex, their position in the circumference of the heart (papillary muscle angle) and the individual's anatomical axis. A standard sized heart and left ventricle were used in each case. It is possible to input more details of the hearts axis, size and myocardial thickness in to the program, also data on the right ventricle can be used. In future studies these additional features can be added in to the program allowing for increased specificity of the ECG produced.

If simultaneously recorded ECGs could be taken this would allow production of the VCG from the original standard ECG data and would mean comparison could be made between vector loops. Frank lead recording could be performed.

This project is still on going and there are several changes that are being made to it and the way the ECG are produce, the amount of information being inputted into the program and the point of excitation initiation.

Bayes de Luna has started to do some work looking at the body type (thin, mid, heavy) and heart axis and it effect on the ECG form. He has been asked to look at this work so all the volunteers have now been classed in body type and their heart axis is to be worked out. To look at the way the heart lies in relation to the ribs is of interest because the electrode positions are decided by the rib positions. If the heart is lying in a position which means the electrodes are not over the expected area of the heart then this may affect the ECG morphology. This information can be worked out from the MRI scan but a landmark in the long axis of the body has to be defined.

\section{References}

[1] Axel L. Papillary muscles do not attach directly to the solid hear wall. Circulation 29 June 2004

[2] Hamilton et al. Human embryology, prenatal development of form and function. William's \& Wilkin co. 567, 1972

[3] Tawara. Tawara's monograph. He conduction system of the mammalian heart 62-66.

[4] Olson CW et al. A dynamic 3D display of the ventricular excitation and generation of the vector and electrocardiogram. J. Electro. 34 sup 2001.

[5] Selvester RH et al. The Selvester scoring system for estimation of myocardial infarction size: The development \& application of the system. Arch Intern Med. 145:1877-1881, 1985.

Name AMC Robinson

Cardiac MRI Research, Phase 4 building, Glasgow Western Infirmary, Glasgow, Scotland.

E-mail address maddyrobinson@yahoo.co.uk 